\title{
Comparación de técnicas de machine learning para la detección de phishing
}

\author{
Andrés Eduardo Moncada-Vargas
}

El phishing es el robo de datos personales a través de una página web falsa donde se le pide al usuario ingresar sus datos para validar su identidad frente a una supuesta entidad legítima. En este trabajo, se compararon técnicas de machine learning y se determinó que la técnica bosque aleatorio es la más efectiva en casos donde las características de las páginas tengan un valor exacto, y árbol de decisión es la más efectiva en casos donde las características hayan sido analizadas y se haya determinado una clasificación en base a dicha característica.

\section{Comparison of Machine Learning Techniques for Phishing Detection}

Phishing is the act of stealing personal data through a false Web page. Users are asked by a supposedly legitimate company to enter their private information in order to verify their identity. This research work compared different machine learning techniques and determined that the random forest technique is the most effective one when the characteristics of the pages have an exact value, and the decision tree is the most effective one when the characteristics of the pages have been analyzed and a classification has been determined based on such characteristics. 


\section{Comparación de técnicas de machine learning para la detección de phishing}

Andrés Eduardo Moncada-Vargas

20152102@aloe.ulima.edu.pe

Resumen: El phishing es el robo de datos personales a través de una página web falsa donde se le pide al usuario ingresar sus datos para validar su identidad frente a una supuesta entidad legítima. En este trabajo se compararon técnicas de machine learning y se determinó que la técnica bosque aleatorio es la más efectiva en los casos en que las características de las páginas tengan un valor exacto y árbol de decisión es la más efectiva en aquellos casos en los cuales las características hayan sido analizadas y se haya determinado una clasificación en base a dicha característica.

\section{Introducción}

El phishing es una de las mayores amenazas en la actualidad, siendo la causa de la mayoría de los robos y estafas cibernéticas en los últimos años. Por dicho motivo, se desarrollaron varias técnicas para detectar páginas phishing. Con el paso del tiempo, los métodos de crear páginas phishing se fueron innovando, por lo que se empezó a usar machine learning para la detección de phishing. En este trabajo se desarrollará una metodología de comparación de técnicas de machine learning para determinar cuál es la mejor en distintos casos. Por ese motivo, se buscará replicar experimentaciones realizadas en el pasado por otros autores, para determinar si los resultados coinciden y realizar una comparación basada en los resultados de otros autores y los obtenidos en esta experimentación.

\section{Materiales y métodos}

Clasificadores de machine learning: árbol de decisión, regresión logística, redes neuronales (perceptrón de multicapas), máquina de soporte vectorial y bosque aleatorio.

Datasets, Phishing BestFirst y Phishing Infogain, obtenidos de la experimentación de Islam et al. (2016), Phishing Legitimate Full, obtenido de la experimentación de Cuzzocrea et al. (2018).

Proceso. Los hiperparámetros de los clasificadores fueron refinados, optimizando los resultados, y se compararon los resultados obtenidos con los vistos en los trabajos de los autores mencionados, para determinar el mejor clasificador en cada caso.

\section{Resultados}

Los resultados obtenidos se muestran

en los siguientes gráficos:

Phishing_BestFirst

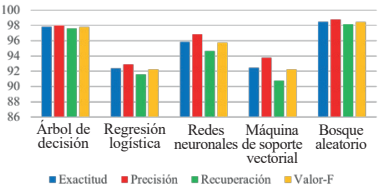

Phishing_Infogain

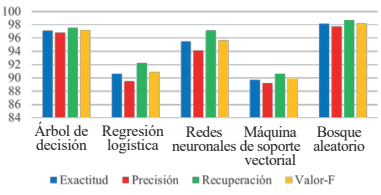

Phishing_Legitimate Full

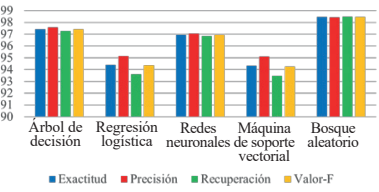

PhishingData

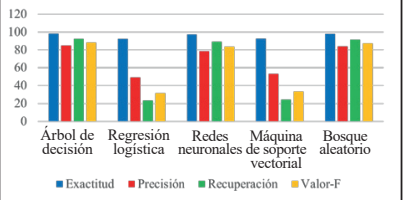

Tras analizar los resultados, se observó lo siguiente:

- Los clasificadores de árboles (árbol de decisión y bosque aleatorio) fueron los más efectivos.

- Bosque aleatorio obtuvo mejores resultados que árbol de decisión con cada dataset, a excepción del último, PhishingData.

- Regresión logística y máquina de soporte vectorial fueron los menos efectivos al momento de clasificar páginas phishing, en comparación con los otros tres clasificadores.

\section{Conclusiones}

Tras investigar técnicas de machine learning y distintos casos de uso, se concluye que los clasificadores de árboles tienen un mejor rendimiento en distintas métricas para la clasificación de páginas phishing. Aunque se ha comprobado que los métodos de aprendizaje automático pueden ser apropiados para la identificación de sitios web phishing, debe considerarse que los procedimientos de análisis deben ser ampliados a nuevos conjuntos de datos para afianzar el universo de casos de uso examinados. En trabajos futuros se espera examinar ese comportamiento.

\begin{tabular}{|ll|}
\hline Referencias & to Detect and Analyze Web \\
Chiew, K. L., Tan, C. L., Wong, K. & Phishing Attacks. ACM Digital \\
S., Yong, K. S., y Tiong, W. K. & Library, 5. \\
(2019). A New Hybrid Ensemble & doi: 10.1145/3282373.3282422 \\
Feature Selection Framework & Islam Mamun,M. S., Rathore, \\
for Machine Learning-Based & M. A., Lashkari, A. H., \\
Phishing Detection System. & Stakhanova, N., y Ghorbani, A. \\
Science Direct, 14. & A. (2016) Detecting Malicious \\
doi: 10.1016/j.ins2019.01.064 & URLs Using Lexical Analysis. \\
Cuzzocrea, A., Martinelli, F., y & Springer Link, 16. \\
Mercaldo, f. (2018). Applying & doi: 10.1007/978-3-319- \\
Machine Learning Techniques & $46298-1$ 30 \\
\hline
\end{tabular}

